\section{Análise preliminar do Sistema de Informação do Programa de Controle da Esquistossomose no período de 1999 a 2003}

\author{
Preliminary analysis of the Information System in \\ the Brazilian Schistosomiasis Control Program, \\ 1999-2003
}

\author{
${ }^{1}$ Escola Nacional de Saúde \\ Pública Sergio Arouca, \\ Fundação Oswaldo Cruz, \\ Rio de Janeiro, Brasil. \\ Correspondência \\ L. M. M. Farias \\ Departamento de Endemias \\ Samuel Pessoa, Escola \\ Nacional de Saúde \\ Pública Sergio Arouca, \\ Fundação Oswaldo Cruz. \\ Rua Leopoldo Bulhões 1480 , \\ Rio de Janeiro, $R J$ \\ 21041-210, Brasil. \\ leilamattos@ensp.fiocruz.br
}

\begin{abstract}
This study provides a preliminary analysis of the Information System in the Schistosomiasis Control Program (SISPCE) in the States of $\mathrm{Ba}$ hia, Sergipe, Alagoas, and Pernambuco, Brazil, from 1999 to 2003. The data analysis suggests a sharp decline in data entry in the system during the transition period involving health services decentralization, followed by a gradual increase. Major under-recording was observed in Pernambuco. SISPCE-DATASUS and IBGE (National Census Bureau) data are insufficient to construct a surveillance index for serious forms of the endemic disease and other aspects that could relate sanitary conditions to schistosomiasis. Reformulation of the SISPCE is key for schistosomiasis control measures, given the new reality in the Brazilian health system. The data system should be redesigned in order to integrate the health policies with the Unified National Health System's guidelines, using a systematic data collection methodology in keeping with local specificities and allowing prospective comparisons and evaluations, in addition to constructing new indicators with an emphasis on morbidity and determinants of the endemic and the adoption of analytical of the that express the real situation of the schistosomiasis in the States.
\end{abstract}

Schistosomiasis; Endemic Diseases; Information Systems
Leila Maria Mattos de Farias 1 Ana Paula da Costa Resendes 1 Paulo Chagastelles Sabroza 1 Reinaldo Souza-Santos 1

\section{Introdução}

A diversidade dos fatores que envolvem a transmissão da esquistossomose dificulta de forma importante o seu controle pelos serviços de saúde 1,2 . As ações de controle da esquistossomose no Brasil vêm sendo adotadas de maneira sistemática e abrangente desde $1976{ }^{3}$. Ao longo das três últimas décadas os programas de controle da esquistossomose (PCE) passaram por diversas fases onde foram feitas tentativas de medicalização maciça dos portadores, conjugadas com outras ações preventivas consideradas importantes 4 .

A informação sobre o comportamento da endemia permite a identificação de problemas individuais e coletivos no quadro sanitário de uma população, propiciando elementos para análise da situação encontrada e subsidiando a busca de possíveis alternativas de encaminhamento, além de abranger ações relativas ao processo saúde/ doença e as de caráter administrativo, essenciais ao processo de tomada de decisão ${ }^{5}$.

Este estudo é uma análise preliminar do Sistema de Informação do Programa de Controle da Esquistossomose (SISPCE), dos Estados da Bahia, Sergipe, Alagoas e Pernambuco, no período pós-descentralização das ações de controle (1999-2003). 


\section{Metodologia}

Foram incluídos no estudo os Estados da Bahia, Sergipe, Alagoas e Pernambuco por representarem áreas de alta transmissibilidade da esquistossomose mansônica 6 .

A análise utilizou dados operacionais gerados durante as atividades de vigilância e controle da esquistossomose, entre 1999 a 2003, originários do SISPCE que está disponível na página de Internet do Departamento de Informação e Informática do SUS (DATASUS: http://www.datasus. gov.br) e o Relatório de Atividades do Projeto de Vigilância e Monitoramento de Endemias do convênio entre a Escola Nacional de Saúde Pública Sergio Arouca, Fundação Oswaldo Cruz (ENSP/ FIOCRUZ) e a Secretaria de Vigilância à Saúde, Ministério da Saúde (SVS/MS) 7,8, baseados em entrevistas realizadas com o Coordenador Nacional e os Coordenadores Regionais do PCE, vinculados à SVS/MS e às Secretarias Estaduais de Saúde, respectivamente.

Inicialmente são apresentados e discutidos os resultados comuns a todos os estados e as particularidades encontradas. Posteriormente são analisados os resultados obtidos com base no banco de dados disponível no SISPCE-DATASUS, para os Estados de Sergipe, Alagoas e Bahia.

Os dados relativos ao Estado de Pernambuco são apresentados separadamente, pois são originários de duas fontes: o SISPCE-DATASUS e dados do sistema local fornecidos pela Gerência Técnica da Esquistossomose, Secretaria Estadual de Saúde que consistem de dados registrados parcialmente pelos municípios.

\section{Resultados e discussão}

A informatização do SISPCE foi iniciada em $1995^{8}$, estando atualmente implantada em todos os estados estudados (Tabela 1). Uma importante alteração ocorrida com a descentralização das ações de controle de endemias, entre 1999 e 2000, se deu na fase de inclusão de dados operacionais no sistema, que anteriormente era executada pelas Coordenações Estaduais do PCE e passou a ser responsabilidade dos municípios 7 .

A distribuição geográfica da endemia atinge cerca de $68 \%$ dos municípios de cada estado estudado, à exceção de Pernambuco onde este porcentual está em torno de 39\% 5 (Tabela 1), concentrada principalmente nas zonas da mata e litorânea 1,6,9

Atualmente a integração das ações de saneamento e as ações de informação, educação, comunicação e mobilização comunitária não estão sendo priorizadas no controle da endemia (Tabela 1). O MS, os estados e os municípios não têm conseguido desenvolver políticas específicas e harmonizadas com as demais ações de controle dessa helmintíase. O mesmo ocorre com a integração com as ações básicas de atenção à saúde, apesar de vinculadas ao Programa Saúde da Família (PSF). A maioria dos municípios não assume as atividades anteriormente do PCE. Em Sergipe, apesar do gestor afirmar que há integração dessas ações no Estado (Tabela 1), não existem indicadores objetivos sobre o trabalho integrado. A baixa adesão das prefeituras ao PSF e o número insuficiente de profissionais para executar as funções atribuídas ao PSF são apontados como causa principal do problema 7 .

Tabela 1

Informações gerais sobre a situação das ações de controle da esquistossomose mansônica no período pós-descentralização (1999-2002)

Bahia, Sergipe, Alagoas e Pernambuco, Brasil.

\begin{tabular}{|c|c|c|c|c|c|c|c|c|c|}
\hline Estado & Total & $\begin{array}{l}\text { Municípios } \\
\text { endêmicos }\end{array}$ & $\%$ & $\begin{array}{c}\text { Sistema de } \\
\text { informação } \\
\text { informatizado }\end{array}$ & $\begin{array}{l}\text { Segue } \\
\text { diretrizes } \\
\text { do MS }\end{array}$ & $\begin{array}{l}\text { Integração } \\
\text { com o PSF de } \\
\text { saneamento }\end{array}$ & $\begin{array}{l}\text { Integração } \\
\text { com ações } \\
\text { em saúde }\end{array}$ & $\begin{array}{c}\text { Integração } \\
\text { com ações } \\
\text { de educação } \\
\text { em saúde }\end{array}$ & $\begin{array}{c}\text { Freqüência } \\
\text { de exames } \\
\text { realizados }\end{array}$ \\
\hline Bahia & 417 & 280 & 67,15 & Sim & Sim & Não & Não & Não & $1 \mathrm{vez}$ ao ano \\
\hline Sergipe & 75 & 51 & 68,00 & Sim & Sim & Sim & Não & Sim & $1 \mathrm{vez}$ ao ano \\
\hline Alagoas & 102 & 70 & 68,63 & Sim & Sim & Não & Não & Não & $1 \mathrm{vez}$ ao ano \\
\hline Pernambuco & 185 & 72 & 38,92 & Sim & Sim & Não & Não & Não & $1 \mathrm{vez}$ ao ano \\
\hline
\end{tabular}

MS = Ministério da Saúde; PSF = Programa Saúde da Família

Fonte: Relatório de Atividades do Projeto de Vigilância e Monitoramento de Endemias 7. 
Com a descentralização, as equipes estaduais são responsáveis pela capacitação dos municípios nas atividades do PCE, inclusive aquelas relacionadas à inclusão dos dados no sistema. A partir de então, são consolidados pelos estados e posteriormente encaminhados para a Gerência Técnica da Esquistossomose da SVS/MS. Contudo, os municípios convivem com alta rotatividade do seu quadro de pessoal responsável pelo registro no SISPCE, que é geralmente deslocado de função, sendo substituído rotineiramente por pessoal não capacitado para aquela atividade 7 .

Não há periodicidade na realização dos exames coproscópicos e registro dos dados. Cada município executa essa atividade de acordo com critérios próprios, dependendo de disponibilidade de equipamentos e de pessoal, que freqüentemente são deslocados para o controle de outras endemias, variando também a metodologia adotada, podendo ser inquérito censitário, levantamento em escolares, ou resultado dos exames decorrentes das demandas locais dos serviços 7,10,11.

Os dados disponíveis no DATASUS (Tabela 2) de 1999 a 2003, indicam que o registro no SISPCE sofreu acentuada queda entre 1999 e 2000, comparando-se aos anos anteriores, coincidindo com a intensificação da descentralização das ações, em conseqüência da municipalização da etapa de realização de exames coproscópicos e posterior informação dos resultados. Com efeito, em Sergipe, dos 51 municípios considerados endêmicos, só há registros em cerca de $18 \%$ desses municípios em 1999 e 23\% em 2000. Na Bahia esses registros apresentaram os mais baixos porcentuais em 1999, cerca de $0,71 \%$, e em 2000 , cerca de 5\%. Alagoas manteve os melhores níveis de informação durante o período de descentralização.

O aumento progressivo de registros (Tabela 2) coincide com a reestruturação das atividades de controle nos níveis estadual e municipal.

Dados do programa de controle da esquistossomose nos Estados de Sergipe, Bahia e Alagoas, Brasil, 1999-2003.

\begin{tabular}{|c|c|c|c|c|c|}
\hline Estado & 1999 & 2000 & 2001 & 2002 & 2003 \\
\hline \multicolumn{6}{|l|}{ Sergipe } \\
\hline Municípios trabalhados & 9 & 17 & 20 & 18 & 28 \\
\hline População trabalhada & 76.623 & 51.655 & 61.828 & 86.958 & 87.030 \\
\hline Exames realizados & 64.002 & 43.703 & 51.622 & 68.310 & 71.379 \\
\hline Indivíduos positivos & 11.020 & 6.201 & 6.107 & 7.356 & 7.575 \\
\hline Prevalência nos examinados (\%) & 17,22 & 14,19 & 11,83 & 10,77 & 10,61 \\
\hline Pessoas tratadas & 9.891 & 5.374 & 5.037 & 5.458 & 6.275 \\
\hline Positivos com alta carga parasitária & 1.264 & 501 & 316 & 255 & 411 \\
\hline \multicolumn{6}{|l|}{ Bahia } \\
\hline Municípios trabalhados & 2 & 15 & 87 & 146 & 127 \\
\hline População trabalhada & 39.221 & 18.826 & 412.831 & 938.360 & 690.540 \\
\hline Exames realizados & 26.482 & 14.913 & 334.892 & 733.805 & 538.201 \\
\hline Indivíduos positivos & 2.920 & 1.582 & 20.196 & 43.154 & 28.232 \\
\hline Prevalência nos examinados (\%) & 11,03 & 10,61 & 6,03 & 5,88 & 5,24 \\
\hline Pessoas tratadas & 2.732 & 1.482 & 18.453 & 38.839 & 24.239 \\
\hline Positivos com alta carga parasitária & 44 & 195 & 1.361 & 2.061 & 666 \\
\hline \multicolumn{6}{|l|}{ Alagoas } \\
\hline Municípios trabalhados & 42 & 30 & 34 & 46 & 59 \\
\hline População trabalhada & 304.892 & 186.372 & 142.141 & 250.995 & 285.442 \\
\hline Exames realizados & 234.223 & 137.349 & 107.743 & 188.467 & 213.374 \\
\hline Indivíduos positivos & 35.156 & 17.230 & 17.773 & 24.272 & 28631 \\
\hline Prevalência nos examinados (\%) & 15,01 & 12,54 & 16,46 & 12,88 & 13,42 \\
\hline Pessoas tratadas & 23.293 & 13.684 & 14.837 & 21.203 & 24.575 \\
\hline Positivos com alta carga parasitária & 3.705 & 1.228 & 1.227 & 1.360 & 2.095 \\
\hline
\end{tabular}

Fonte: Sistema de Informação do Programa de Controle da Esquistossomose.

Obs. Os dados ainda são parciais (existem informações de campo para serem digitadas no SISPCE), à exceção do Estado de Alagoas que concluiu a digitação até o ano de 2002. 
Porém, a sua insuficiência e a indefinição de metodologia, que contemple todas as localidades das áreas endêmicas com periodicidade definida, impossibilitam análise mais fidedigna da atual situação da esquistossomose.

Em Pernambuco (Tabela 3) o total de registros do SISPCE é bastante inferior aos apresentados como dados do sistema local, e também, ao que foi efetivamente realizado no estado, indicando um sub-registro no sistema. Por outro lado, nos sistemas locais, os dados de 2001 apresentam importante discrepância em relação aos demais anos, sugerindo engano na digitação.

Os dados disponíveis até o momento para os estados analisados, indicam taxas de prevalência no período de 2000 a 2003 bem inferiores às encontradas na década 70 , quando foram iniciadas as atividades do PCE no Nordeste ${ }^{12}$, porém com porcentuais praticamente estáveis e acima do estabelecido para que a endemia entre no estágio de vigilância.

A base de dados atualmente disponível permite o acesso ao registro do número de indivíduos com alta carga parasitária, sendo este um importante dado para a construção de indicador com vistas a monitorar a magnitude da endemia e fornecer elementos para a programação local de ações para o controle da helmintíase. No entanto, ainda não há integração das informações sobre a infecção com aquelas disponíveis no Sistema de Informações Hospitalares (SIH), responsável pelo registro de formas clínicas graves. O mesmo fenômeno ocorre com os indicadores geralmente utilizados para analisar a relação da doença com a disponibilidade de água potável e esgotamento sanitário.

\section{Conclusão}

Atualmente o SISPCE reflete a forma como foi estruturado o controle das endemias nos municípios, originário da estrutura centralizada e verticalizada que sempre pautou os PCE em décadas passadas, contribuindo apenas com dados operacionais e agregando poucas informações que se traduzam em indicadores epidemiológicos para o efetivo controle e vigilância da esquistossomose.

A desestruturação das ações no período pós-descentralização concorreu de forma importante para a deficiência na etapa de registro dos dados no sistema, que se expressa pela inconsistência dos dados e irregularidade do seu registro. Isso implicou dificuldades significativas na avaliação das ações de controle pautada em sistema de informações inadequado para a atual estrutura do sistema de saúde (Sistema Único de Saúde-SUS).

A reformulação do sistema de informações é etapa fundamental para a efetivação das ações de controle diante da nova realidade do sistema de saúde. Esse deve ser repensado de forma a integrar-se às políticas de saúde em consonância com as diretrizes do SUS, com a observância de metodologia de coleta de dados sistemática, adequada às especificidades locais, possibilitando comparações e avaliações prospectivas, além da construção de novos indicadores com ênfase na morbidade e nos determinantes da endemia e da adoção de unidades de análise que venham a expressar a real situação da esquistossomose nos estados.

Dados relativos às atividades do Programa de Controle da Esquistossomose (PCE) no Estado de Pernambuco, Brasil, segundo Sistema de Informação do Programa de Controle da Esquistossomose (SISPCE) e dados dos sistemas locais.

\begin{tabular}{|c|c|c|c|c|c|c|c|c|c|c|}
\hline \multirow[b]{2}{*}{$\begin{array}{l}\text { Atividades } \\
\text { do PCE }\end{array}$} & \multicolumn{2}{|c|}{1999} & \multicolumn{2}{|c|}{2000} & \multicolumn{2}{|c|}{2001} & \multicolumn{2}{|c|}{2002} & \multicolumn{2}{|c|}{2003} \\
\hline & SISPCE & $\begin{array}{c}\text { Dados do } \\
\text { sistema } \\
\text { local }\end{array}$ & SISPCE & $\begin{array}{c}\text { Dados do } \\
\text { sistema } \\
\text { local }\end{array}$ & SISPCE & $\begin{array}{c}\text { Dados do } \\
\text { sistema } \\
\text { local }\end{array}$ & SISPCE & $\begin{array}{c}\text { Dados do } \\
\text { sistema } \\
\text { local }\end{array}$ & SISPCE & $\begin{array}{c}\text { Dados do } \\
\text { sistema } \\
\text { local }\end{array}$ \\
\hline \multicolumn{11}{|l|}{ Municípios } \\
\hline trabalhados & * & * & * & * & * & * & * & * & * & * \\
\hline Exames realizados & * & 111.163 & 2.369 & 78.878 & 30.741 & 11.153 & 38.950 & 112.253 & 73.436 & 115.923 \\
\hline Pessoas positivas & * & 11.830 & 262 & 6.722 & 4.069 & 11.123 & 3.853 & 8.960 & 6.729 & 9.340 \\
\hline \multicolumn{11}{|l|}{ Prevalência nos } \\
\hline examinados (\%) & * & 10,64 & 11,06 & 8,52 & 13,24 & 99,73 & 9,89 & 7,98 & 9,16 & 8,05 \\
\hline Pessoas tratadas & * & * & 341 & * & 3.385 & * & 3.275 & * & 5.463 & * \\
\hline
\end{tabular}

\footnotetext{
* Sem registro.
}

Fonte : SISPCE-DATASUS e dados locais fornecidos pela Coordenação Estadual do PCE de Pernambuco (agosto/2004). 


\section{Resumo}

O estudo teve como objetivo a análise preliminar do Sistema de Informação do Programa de Controle da Esquistossomose (SISPCE), na Bahia, Sergipe, Alagoas e Pernambuco, Brasil, entre 1999 e 2003. Houve queda acentuada da entrada de dados no período da descentralização, com aumento gradativo dos registros, à exceção de Pernambuco que apresenta um sub-registro importante. Os dados do SISPCE-DATASUS e IBGE são insuficientes para construção de indicadores para o monitoramento das formas graves da endemia e outros aspectos que possam relacionar as condições de saneamento com a ocorrência da doença. A reformulação do sistema de informações é fundamental para a efetivação das ações de controle diante da nova realidade do sistema de saúde. Esse deve ser repensado de forma a integrar-se às políticas de saúde em consonância com as diretrizes do SUS, utilizando metodologia de coleta de dados sistemática, adequada às especificidades locais, possibilitando comparações e avaliações prospectivas, além da construção de novos indicadores com ênfase na morbidade e nos determinantes da endemia e da adoção de unidades de análise que venham a expressar a real situação da esquistossomose nos Estados.

Esquistossomose; Doenças Endêmicas; Sistemas de Informação

\section{Colaboradores}

L. M. M. Farias participou de todas as fases da pesquisa e redação deste trabalho. A. P. C. Resendes contribuiu na construção dos indicadores. P. C. Sabroza participou do planejamento, discussão dos resultados e revisão crítica do manuscrito. R. Souza-Santos contribuiu na discussão dos resultados e revisão crítica do manuscrito.

\section{Agradecimentos}

A Hélio Yamada pela disponibilização dos dados.

\section{Referências}

1. Resendes APC, Souza-Santos R, Barbosa CS. Internação hospitalar e mortalidade por esquistossomose mansônica no Estado de Pernambuco, Brasil, 1999/2000. Cad Saúde Pública 2005; 21:1392-401.

2. Coura-Filho P. Participação popular no controle da esquistossomose através do Sistema Único de Saúde (SUS), em Taquaruçu de Minas, (Minas Gerais, Brasil), entre 1985-1995: construção de um modelo alternativo. Cad Saúde Pública 1998; 14 Suppl 2:111-22.

3. Carmo EH, Barreto ML. Esquistossomose mansônica no Estado da Bahia, Brasil: tendências históricas e medidas de controle. Cad Saúde Pública 1994; 10:425-39.

4. Amaral RS, Porto MAS. Evolução e situação do controle da esquistossomose no Brasil. Rev Soc Bras Med Trop 1994; 27:73-90.

5. Branco MAF. Sistemas de informação em saúde no nível local. Cad de Saúde Pública 1996; 12:267-70.

6. Noronha CV, Barreto ML, Silva TM, Souza IM. Uma concepção popular sobre a esquistossomose mansônica: os modos de transmissão e prevenção na perspectiva de gênero. Cad Saúde Pública 1995; 11:106-17.

7. Ministério de Saúde. Relatório de atividades do projeto de vigilância e monitoramento de endemias do convênio entre ENSP/FIOCRUZ e SVS/MS. Brasília: Secretaria de Vigilância em Saúde, Ministério de Saúde; 2005.

8. Secretaria de Vigilância em Saúde, Ministério da Saúde. Vigilância epidemiológica: PCE - Programa de Controle da Esquistossomose. http://dtr2001. saude.gov.br/svs/epi/esquis/esquis00.htm (acessado em 13/Fev/2006).

9. Silva LJ. A esquistossomose mansônica no Estado de São Paulo: origens, distribuição, epidemiologia e controle [Tese de Livre Docência]. Campinas: Faculdade de Ciências Médicas, Universidade Estadual de Campinas; 1992.

10. Barbosa CS, Silva CB, Barbosa FS. Esquistossomose: reprodução e expansão da endemia no Estado de Pernambuco no Brasil. Rev Saúde Pública 1996; 30:609-16.

11. Favre TC, Pieri OS, Barbosa CSB, Beck L. Avaliação das ações de controle da esquistossomose implementadas entre 1977 e 1996 na área endêmica de Pernambuco, Brasil. Rev Soc Bras Med Trop 2001; 34:569-76.

12. Coura JR, Amaral RS. Epidemiological and control aspects of schistosomiasis in Brazilian endemic areas. Mem Inst Oswaldo Cruz 2004; 99 Suppl 1: 13-9.

Recebido em 23/Mai/2006

Versão final reapresentada em 28/Ago/2006

Aprovado em 30/Ago/2006 